\title{
Ion Mobility Shift of Isotopologues in a High Kinetic Energy Ion Mobility Spectrometer (HiKE-IMS) at Elevated Effective Temperatures
}

\author{
Christoph Schaefer*, Ansgar T. Kirk, Maria Allers, Stefan Zimmermann \\ Leibniz University Hannover, Institute of Electrical Engineering and Measurement Technology, Department of Sensors and \\ Measurement Technology, Appelstr. 9A, 30167 Hannover, Germany \\ *Corresponding author: schaefer@geml.uni-hannover.de
}

Keywords: ion mobility, isotopologues, effective temperature, ion mobility spectrometry, high kinetic energy ion mobility spectrometry, HiKE-IMS, IMS

\begin{abstract}
Ion mobility spectrometers (IMS) separate ions mainly by ion-neutral collision cross section and to a lesser extent by ion mass and effective temperature. When investigating isotopologues, the difference in collision cross section can be assumed negligible. Since the mobility shift of isotopologues is thus mainly caused by their difference in mass and effective temperature the investigation of isotopologues can provide important insights into the theory of ion mobility. However, in classical IMS operated at ambient pressure, cluster formation with neutral molecules occurs, which significantly influences the mobility shift of isotopologues and thus makes a sound investigation of the effect of ion mass and effective temperature on the ion mobility difficult. In this work, the relative ion mobility of several organic compounds and their ${ }^{13} \mathrm{C}$-labelled isotopologues is studied in a High Kinetic Energy Ion Mobility Spectrometer (HiKE-IMS) at high reduced electric fields up to $120 \mathrm{Td}$, which allows the investigation of non-clustered ion species and thus enables a sound investigation of the mobility shift of isotopologues. The results show that the measured relative ion mobilities of isotopologues having the same effective temperature, and thus their ion mass dominating the relative ion mobility, agree well with theoretical relative ion mobilities predicted by the theory of ion mobility.
\end{abstract}

\section{INTRODUCTION}

Ion mobility spectrometers (IMS) separate ions in the gas phase based on their mobility in a neutral gas under the influence of an electric field. In contrast to mass spectrometers (MS), IMS do not separate ions by mass-to-charge ratio, but mainly by ion-neutral collision cross section depending on the ion size and structure ${ }^{1}$. Therefore, IMS are often coupled with MS to obtain an additional separation dimension and additional information about the ions besides the mass-to-charge ratio ${ }^{2,3}$.

By applying an electric voltage to an IMS, the ions are accelerated in the gas phase in the direction of the electric field, whereas they are decelerated by collisions with neutral molecules. As a result, the ions drift with an average drift velocity $v_{\mathrm{D}}$ along the electric field $E$ according to eq. 1 , where $K_{0}$ is the reduced ion mobility, $\mathrm{N}_{0}$ is the Lohschmidt constant and $N$ is the number of neutral gas molecules per volume. The drift velocity is thus proportional to the quotient $E / N$, the so-called reduced electric field strength, which is usually given in the unit Townsend $\left(1 \mathrm{Td}=1 \cdot 10^{-17} \mathrm{~V} \cdot \mathrm{cm}^{2}\right)$. Therefore, the reduced electric field is a measure for the kinetic energy of the ions and is thus an important quantity in ion mobility experiments.

$$
v_{D}=K_{0} \mathrm{~N}_{0} \frac{E}{N}
$$

The reduced ion mobility $K_{0}$ from eq. 1 is given by the well-known eq. 2. Originally, this theory goes back to the work of Langevin ${ }^{4}$, who obtained a relationship for the mobility of ions in weak electric fields. More detailed studies on ion mobility theory can be found in the work of Mason and $\mathrm{McDaniel}^{5}$ and in a recently published work of Viehland ${ }^{6}$. In eq. 2, $z$ denotes the charge state of the ion, e the elementary charge, $\mathrm{k}_{\mathrm{B}}$ the Boltzmann constant, $T_{\text {eff }}$ the effective temperature of the ion, and $\Omega$ the ion-neutral collision cross section. In addition, $\mu=\left(m^{-1}+M^{-1}\right)^{-1}$ is the reduced mass of the ion-neutral pair, where $m$ is the mass of the ion and $M$ is the mass of the neutral molecule. It should be noted, that eq. 2 is an approximation assuming atomic ions in an atomic neutral gas that in some cases can have an error of $5 \%$ or even more depending on ion-neutral mass ratios and ion-neutral interaction potentials ${ }^{5}$. However, the resulting correction factor $\alpha$ is often negligible ${ }^{7,8}$.

$$
K_{0}=\frac{3}{16} \frac{z \mathrm{e}}{\mathrm{N}_{0}} \sqrt{\frac{2 \pi}{\mu \mathrm{k}_{\mathrm{B}} T_{\text {eff }}}} \frac{(1+\alpha)}{\Omega}
$$

In eq. 2, the effective temperature of the ion, i.e. the average ion-neutral collision energy in the center-of-mass frame of the ions and neutral molecules, is used instead of the commonly used absolute temperature $T$. This considers that the energy of the ions can differ significantly from their thermal energy. This expression was derived by Viehland and Mason to modify the mobility equation to be applicable at arbitrary field strengths ${ }^{7}$. 
The ion-neutral collision cross section $\Omega$ in eq. 2 is derived by several integrations averaging the ion-neutral collisions over scattering angles and energies and thus correlates with the size and structure of the ions and their interaction with the neutral molecules ${ }^{9}$. The ion mobility of different ion species differs mainly due to differences in their collision cross sections and to a lesser extent due to different effective temperatures and ion masses. However, if the usually small correction factor $\alpha$ is neglected, the ion mass only influences the reduced mass term, which in turn is always dominated by the smaller mass. If the mass of the ion is in the same order of magnitude as the mass of the neutral molecules, the reduced mass is strongly influenced by both the ion and the neutral molecule. In this case, the ion mobility depends on the ion mass, effective temperature and collision cross section. However, if the ion mass is large compared to the mass of the neutral molecules, the reduced mass is nearly independent of the ion mass, so that the ion mobility just depends on the effective temperature and on the collision cross section. Usually the latter case occurs when conducting ion mobility experiments, since often polyatomic ions are studied in comparatively light drift gases such as nitrogen, helium and purified air ${ }^{10,11}$. Therefore, the ion mobility and thus the separation usually depends rather on the size and structure of the ions than on their mass difference. Furthermore, the ion-neutral collision cross section as well as the ion mass depend on the effective temperature, since ions can form clusters with neutral molecules at low effective temperatures ${ }^{12}$, which in turn can dissociate at elevated effective temperatures ${ }^{13,14}$. The reduced ion mobility increases due to cluster dissociation mainly due to the decreased collision cross section and to a lesser extent due to the decreased ion mass.

In order to separate different ion species in an IMS, their ion mobilities must sufficiently differ depending on the IMS resolving power. A measure for the mobility difference or mobility shift between two ions is their relative ion mobility $K_{\mathrm{r}}$, which is given for two ion species in a neat drift gas by eq. 3. This equation can easily be derived by forming the quotient of the reduced ion mobilities of two ion species according to eq. 2 .

$$
K_{\mathrm{r}}=\frac{K_{0, \mathrm{~A}}}{K_{0, \mathrm{~B}}}=\sqrt{\frac{\mu_{\mathrm{B}}}{\mu_{\mathrm{A}}} \frac{T_{\mathrm{eff}, \mathrm{B}}}{T_{\mathrm{eff}, \mathrm{A}}} \frac{\Omega_{\mathrm{B}}}{\Omega_{\mathrm{A}}}}
$$

As can be seen, the relative ion mobility should only depend on the reduced mass of the ions and the neutral molecules, their respective ion-neutral collision cross sections and on their effective temperatures. The latter is given by eq. 4, which is the well-known Wannier equation $^{15}$. The term $T$ of eq. 4 corresponds to the thermal energy of the ion, and the term $T_{\text {field }}$ corresponds to the energy the ion acquires from the electric field. At low reduced electric fields, $T_{\text {field }}$ should be negligible, so that the ion energy is approximately equal to the thermal ion energy $T$. In this case, ions of two different species should have approximately the same effective temperature. However, $T_{\text {field }}$ can no longer be neglected at high reduced electric fields, since it can reach about $T_{\text {field }}=750 \mathrm{~K}$ in nitrogen with $M=28 \mathrm{u}$ at a reduced electric field of $E / N=120 \mathrm{Td}$ for ions with a reduced ion mobility of $K_{0}=2.5 \mathrm{~cm}^{2} / \mathrm{Vs}$. In this case, the energy the ion acquires from the electric field predominates over its thermal energy. $T_{\text {field }}$ differs for different ion species due to different reduced ion mobilities $K_{0}$, resulting in different effective temperatures at high reduced electric fields. This difference strongly depends on the reduced electric field strength due to the quadratic appearance of $E / N$ in eq. 4 and must therefore be considered when investigating the relative ion mobility of two ions at elevated reduced electric fields. The correction factor $\beta$ depends on the masses of the ions and neutrals and on their interaction potential, similar to the correction factor $\alpha$ in eq. 2 . The correction factor $\beta$ is usually also negligible ${ }^{7,8}$.

$$
T_{\text {eff }}=T+T_{\text {field }}=T+\frac{M v_{\mathrm{D}}^{2}}{3 \mathrm{k}_{\mathrm{B}}}(1+\beta)=T+\frac{M}{3 \mathrm{k}_{\mathrm{B}}}\left(K_{0} N_{0} \frac{E}{N}\right)^{2}(1+\beta)
$$

In addition to the translational degrees of freedom, internal energy states must be considered when investigating polyatomic ions and neutrals. Like the translational energy of the neutral molecules, the internal energy states are characterized by the thermal energy $T$. Since $T_{\text {field }}$ of the ions significantly exceeds the thermal energy at high reduced electric fields, the ions transfer an average energy to the internal degrees of freedom of the neutrals by inelastic collisions ${ }^{8}$. The effective temperature can be significantly affected by this energy loss due to inelastic collisions at high reduced electric fields. With eq. 5, a generalized form of the Wannier equation was introduced by Viehland et al. ${ }^{16}$, where the dimensionless ratio of the collision integral for inelastic energy loss to that for momentum transfer $\xi$ accounts for the fractional energy loss due to inelastic collisions. The ratio $M / m$ considers that the energy loss is distributed unequally between ions and neutrals depending on their masses. This causes an additional discrepancy in the effective temperatures of two different ion species due to different ion masses $m$ if $\xi \neq 0$.

$$
T_{\text {eff }}^{\prime}=\left[1+\frac{M}{m} \xi\right]^{-1} T_{\text {eff }}
$$

In this context, isotopologues are interesting molecules, since the difference in their collision cross section can be assumed negligible, except for vibrational effects occurring when hydrogen is exchanged by deuterium ${ }^{17}$. The relative ion mobility between such ions should therefore only depend on the reduced mass of the ions and neutral molecules, and additionally on their effective temperatures at high reduced electric fields. Furthermore, the investigation of the ratio of the reduced ion mobilities of isotopologues reduces the influence of the error of eq. 2. Hence, investigating the relative ion mobility of isotopologues is useful to validate the theory of ion mobility describing the motion of ions in electric fields. If valid, this theory must be able to explain the observed relative ion mobilities. Such studies could also provide insights into the influence of the effective temperature on the reduced ion mobility. In addition, since the relative ion mobility of isotopologues should only depend on their reduced masses at low reduced electric fields, the relative ion mobility would allow direct mass measurements if the mass difference of the ions is known ${ }^{18,19}$. Furthermore, understanding the relative ion mobility of isotopologues would allow for using isotopically labelled substances as markers or internal standards in ion mobility experiments.

Since isotopologues should have nearly the same collision cross sections, their relative ion mobility, i.e. the ratio of reduced ion mobilities, should be quite small. Since high resolving power is required to separate the isotopologues, such studies have rarely been reported so far. Nevertheless, isotopologues have already been separated in an ultra-high resolving power ion mobility spectrometer with a resolving power of $R_{\mathrm{p}}=250^{18,20}$ operated at ambient pressure. However, due to the low effective temperatures at ambient pressure, ions can form clusters with neutral molecules. This cluster formation can have a significant influence on the relative ion mobility, as the relative ion mobility should decrease due to the decreasing difference in the reduced masses. Consequently, the relative ion mobility would not agree with the theoretical relative ion mobilities calculated with eq. 3 . 
Isotopomers ${ }^{21}$ and isotopologues ${ }^{22,23}$ were also separated in a field asymmetric ion mobility spectrometer (FAIMS). However, in these devices the difference in ion mobility between high and low electric fields is investigated, but not the reduced ion mobility itself. The separation of isotopologues is therefore not based on different reduced masses of the ions and neutral molecules, but rather on nonlinear effects, e.g. cluster formation and dissociation, and is also affected by the energetics of the ion-molecule collisions ${ }^{24}$. Furthermore, isotopologues have different effective temperatures at the high electric fields present in FAIMS. In addition, the center of ion mass is shifted when substituting atoms by their isotopes, which may possibly lead to a changed partition of energy in rotational and translational degrees of freedom in collisions at high kinetic energies ${ }^{25}$, possibly also affecting the effective temperatures. In addition, Wojcik et al. investigated the relative ion mobility of isotopologues in their SLIM SUPER IMS ${ }^{26}$ in helium at low effective temperatures. They observed an additional mobility shift resulting from small differences in the collision cross sections, which however should be negligible in this work compared to the mobility shift caused by different reduced masses as these experiments are conducted in nitrogen.

In this work, we investigate the relative ion mobility of benzene, toluene, acetone and acetonitrile and their ${ }^{13} \mathrm{C}$-labelled isotopologues in a High Kinetic Energy Ion Mobility Spectrometer (HiKE-IMS) in nitrogen. The device is operated at a reduced pressure of 20 mbar, thus allowing the operation at high reduced electric fields up to $E / N=120 \mathrm{Td}$. As shown previously ${ }^{27}$, clusters of ions with neutral molecules should dissociate in the HiKE-IMS due to their high effective temperatures. The investigation of non-clustered isotopologues enables a proper comparison of the observed and theoretical relative ion mobilities. In particular, the investigation of the relative ion mobilities of isotopologues at high reduced field strengths has not been reported so far. It is worth mentioning, that benzene, toluene, acetone and acetonitrile are just model substances for investigating the relative ion mobility of isotopologues. The focus of this paper is not on the detection of the investigated substances by HiKE-IMS but to experimentally investigate the academic question of the ion mobility shift of isotopologues. We specifically selected these substances since they are easy to obtain and their differences in mass and thus in ion mobility are large enough to separate them with the current resolving power of HiKE-IMS. In addition, benzene and toluene form two different stable product ion species via charge transfer and proton transfer, respectively, providing additional experimental data and thus possibly additional insights.

\section{EXPERIMENTAL}

For all experiments, the HiKE-IMS was used, whose current setup was described in detail in an earlier publication ${ }^{28}$. The most important operating parameters are summarized in Table 1 . In order to identify the detected ion species a HiKE-IMS-MS was used ${ }^{27}$. The reduced field strength in both, the reaction and drift region, can be independently increased up to $120 \mathrm{Td}$. The reduced reaction field influences the reactant ion and product ion formation, as discussed in detail in earlier publications ${ }^{27,29,30}$. The reduced drift field influences the separation of different ion species due to the influence of field dependent cluster formation and dissociation on reduced ion mobilities and due to the dependence of reduced ion mobilities on effective temperature.

Table 1. Operating parameters of the HiKE-IMS

\begin{tabular}{ll}
\multicolumn{1}{c}{ Parameter } & \multicolumn{1}{c}{ value } \\
reaction region length & $77 \mathrm{~mm}$ \\
drift region length & $306 \mathrm{~mm}$ \\
drift region diameter & $21 \mathrm{~mm}$ \\
corona voltage & $1250 \mathrm{~V}$ \\
reaction region voltage & $0.5-5 \mathrm{kV}$ \\
reduced reaction field, $E_{\mathrm{RT}} / N$ & $10-120 \mathrm{Td}$ \\
drift region voltage & $0-20 \mathrm{kV}$ \\
reduced drift field, $E_{\mathrm{DT}} / N$ & $0-120 \mathrm{Td}$ \\
injection time & $1 \mu \mathrm{s}$ \\
drift gas flow & $20 \mathrm{ml} / \mathrm{min}$ \\
sample gas flow & $10 \mathrm{ml} / \mathrm{min}$ \\
dew point of sample and drift gas & $-90{ }^{\circ} \mathrm{C}$ \\
operating pressure & $20 \mathrm{mbar}$ \\
operating temperature & $45^{\circ} \mathrm{C}$
\end{tabular}

All chemicals were purchased from Sigma-Aldrich Germany with a purity $>99 \%$. The samples were introduced using a Vici Dynacalibrator Model 150 permeation oven and homemade permeation tubes. All measurements were carried out using nitrogen as drift gas. Drift gas is supplied using an F-200DV-ABD mass flow controller (Bronkhorst, Germany). Sample gas is connected to the HiKE-IMS via a $750 \mu \mathrm{m}$ inner diameter capillary, whose length has been adjusted to provide the sample flow rate of $10 \mathrm{ml} / \mathrm{min}$ (milliliter standard per minute, mass flow at reference conditions $20^{\circ} \mathrm{C}$ and $1013.25 \mathrm{hPa}$ ). To minimize cluster formation with water molecules, the dew point of the sample gas and drift gas was kept low at $-90{ }^{\circ} \mathrm{C}$ using a pressure swing absorber and two moisture traps.

\section{RESULTS \& DISCUSSION}

In this work, toluene, benzene, acetone, acetonitrile and their isotopologues are investigated at various reduced drift fields of $E_{\mathrm{DT}} / N=40-120 \mathrm{Td}$ by simply sweeping the drift voltage and keeping the pressure and thus the density of neutral molecules constant. The reduced reaction field was set to $E_{\mathrm{RT}} / N=70 \mathrm{Td}$ to ensure that all substances, including benzene and toluene, can be ionized. Furthermore, at such reduced reaction field significant fragmentation of product ions in the reaction tube can be avoided.

Figure 1 shows the ion mobility spectrum of toluene and toluene- ${ }^{13} \mathrm{C}_{7}$ in nitrogen at a reduced drift field of $E_{\mathrm{DT}} / N=110 \mathrm{Td}$ as an example. The ion mobility spectra of all other investigated substances, including a blank spectrum without any analytes can be found 
in the Supporting Information. The reactant ion peak is not visible in Figure 1, since it has a reduced ion mobility of about $K_{0}=2.7 \mathrm{~cm}^{2} / \mathrm{Vs}$ under these conditions as seen in Figure $\mathrm{S} 1$ in the Supporting Information.

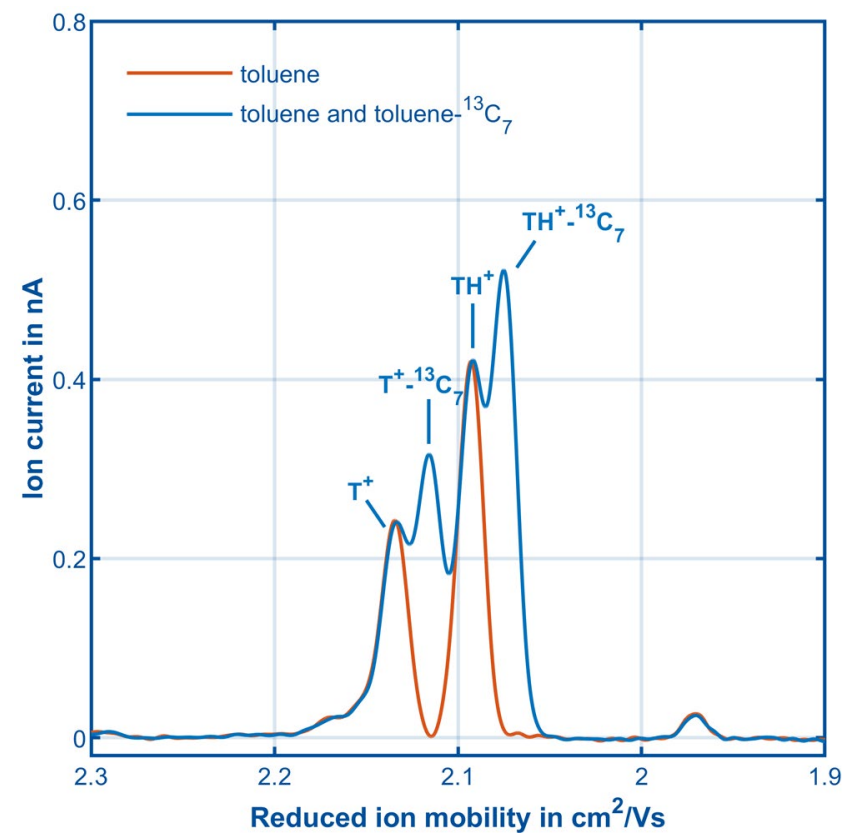

Figure 1. Ion mobility spectrum of $900 \mathrm{ppb}$ v toluene and $1200 \mathrm{ppb}$ v toluene- ${ }^{13} \mathrm{C}_{7}$ in nitrogen at a reduced drift field of $E_{\mathrm{DT}} / N=110 \mathrm{Td}$ and a reduced reaction field of $E_{\mathrm{RT}} / N=70 \mathrm{Td}$. All other experimental parameters are listed in Table 1. Note that the reactant ion peak is not visible on this scaling.

The ion mobility spectrum of toluene and toluene- ${ }^{13} \mathrm{C}_{7}$ shows four peaks, although these peaks are not baseline resolved. If only toluene is measured, just two of the peaks can be detected. The mass spectrum of toluene contained in the Supporting Information shows two peaks with $\mathrm{m} / \mathrm{z}=92$ and $\mathrm{m} / \mathrm{z}=93$, corresponding to the monomer $\mathrm{T}^{+}$directly ionized in a charge transfer reaction and the protonated monomer $\mathrm{TH}^{+}$ionized in a proton transfer reaction. The peak with the higher reduced ion mobility is the directly ionized monomer and the other correspondingly the protonated monomer, since its larger mass and collision cross section cause a smaller reduced ion mobility. The two additional peaks appearing when toluene- ${ }^{13} \mathrm{C}_{7}$ is added are presumably their ${ }^{13} \mathrm{C}$-labelled isotopologues, since their mobility shift regarding the product ions of toluene is quite small. In the following, this will be further validated by investigating their relative ion mobility.

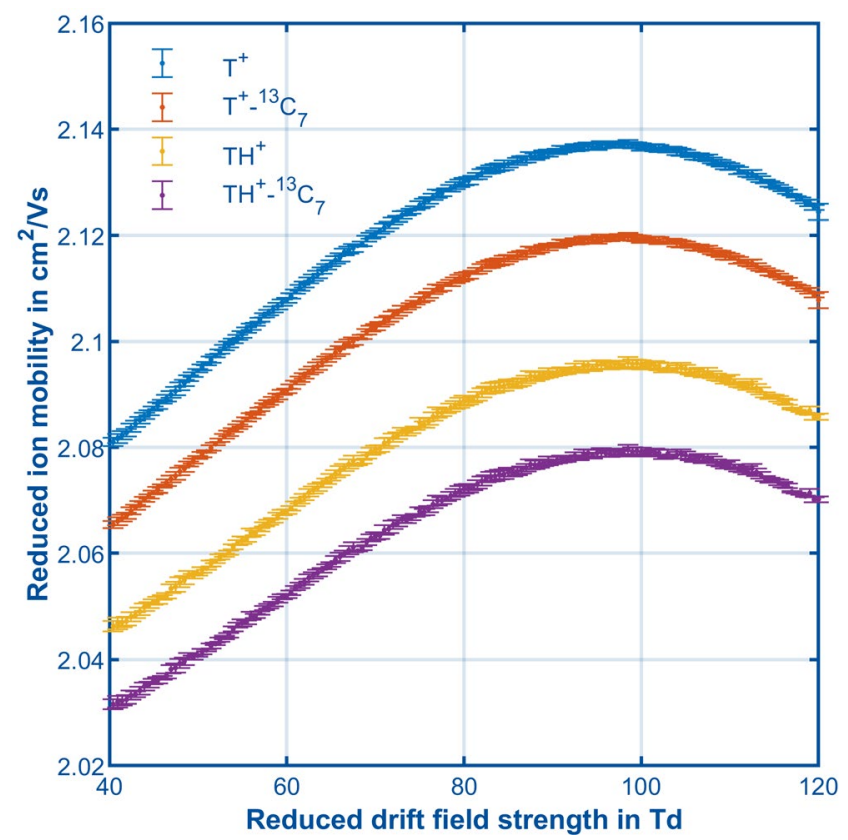

Figure 2. Reduced ion mobilities for the directly ionized and protonated monomer of toluene $\left(\mathrm{T}^{+}\right.$and $\left.\mathrm{TH}^{+}\right)$and for the directly ionized and protonated monomer of toluene- ${ }^{13} \mathrm{C}_{7}\left(\mathrm{~T}^{+}{ }_{-}^{13} \mathrm{C}_{7}\right.$ and $\left.\mathrm{TH}^{+}{ }^{13} \mathrm{C}_{7}\right)$ in nitrogen at various reduced drift fields and a reduced reaction field of $E_{\mathrm{RT}} / N=70 \mathrm{Td}$. All other experimental parameters are listed in Table 1. The error bars represent the standard deviation from three individual measurements of the same sample.

In order to determine the relative ion mobility of the isotopologues, their reduced ion mobilities at all used reduced drift fields have to be determined first. For this purpose, the peaks occurring in the ion mobility spectra are fitted with a Gaussian curve. Both product ions of toluene and toluene- ${ }^{13} \mathrm{C}_{7}$ can be separated from the reactant ion peak (RIP) for all used reduced drift fields, since their reduced ion mobilities sufficiently differ. As can be seen in Figure 2, the reduced ion mobilities of the isotopologues have a similar field 
dependence, as they increase up to a reduced drift field of about $E_{\mathrm{DT}} / N=100 \mathrm{Td}$ until they decrease again at higher reduced drift fields. The increase of $K_{0}$ could be caused by cluster dissociation due to the increasing effective temperatures, similar to what was shown for the reactant ions previously ${ }^{27}$. After all clusters dissociated at high reduced drift fields, a further increase of the effective temperatures causes a decrease of the reduced ion mobility due to its $T_{\mathrm{eff}}^{-1 / 2}$ dependence. In addition, the correction factors $\alpha$ and $\beta$ for equations 2 and 4 are determined according to equations 60 and 61 in ${ }^{7}$. With maximum values of $|\alpha|=0.0041$ and $|\beta|=0.0054$, the correction factors are quite small. Furthermore, since the correction factors for two isotopologues are almost identical, they can be neglected when investigating relative ion mobilities and the differences in the effective temperatures of the isotopologues.
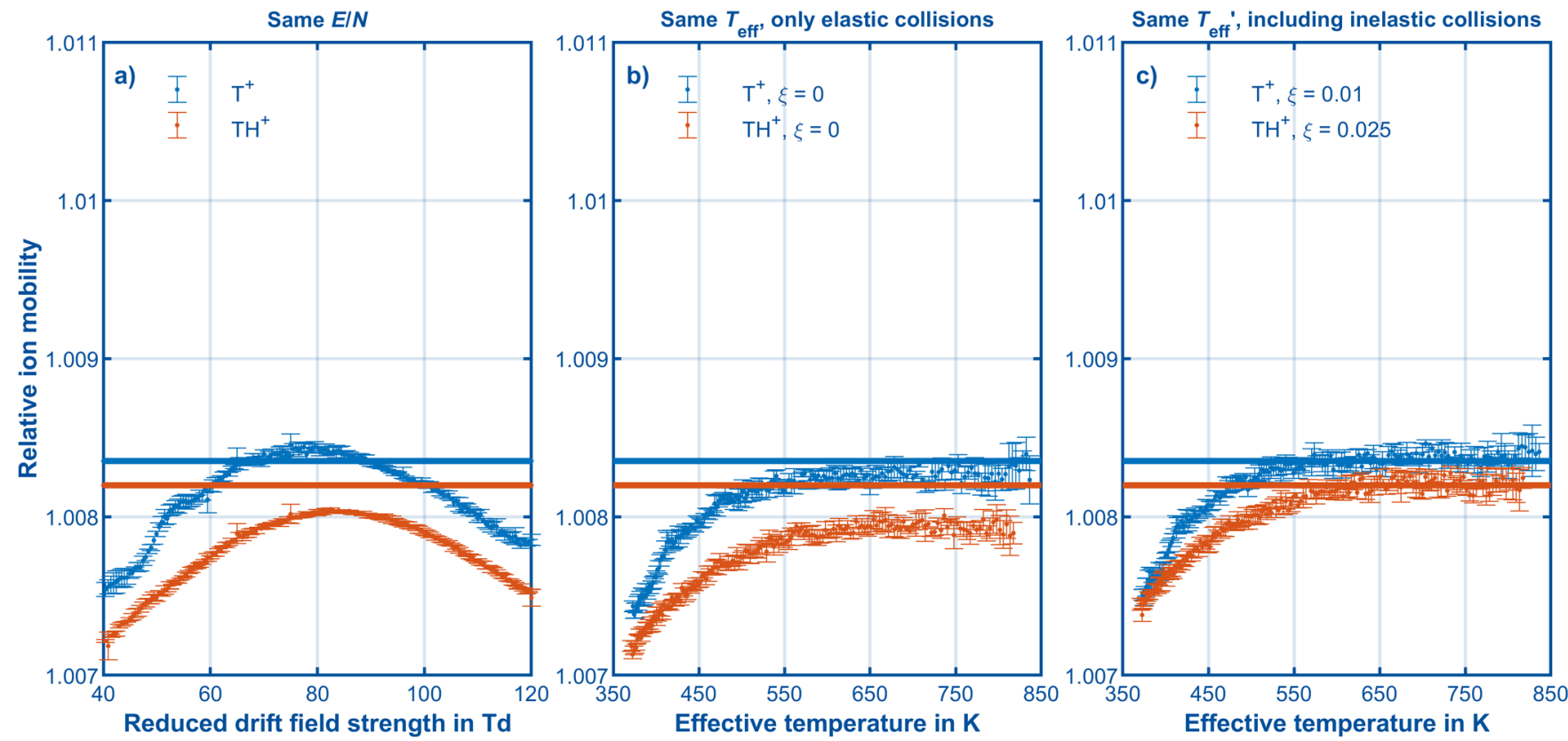

Figure 3. a) Relative ion mobilities $K_{\mathrm{r}}$ at the same reduced drift fields and relative ion mobilities $K_{\mathrm{r}}$ ' at the same effective temperatures b) considering only elastic collisions and c) including inelastic collisions for the directly ionized monomers $\left(\mathrm{T}^{+}\right)$and the protonated monomers $\left(\mathrm{TH}^{+}\right)$of toluene and toluene- ${ }^{13} \mathrm{C}_{7}$ in nitrogen at a reduced reaction field of $E_{\mathrm{RT}} / N=70 \mathrm{Td}$. The relative ion mobilities at the same reduced drift fields are given in dependence of the reduced drift field, which was swept from $E_{\mathrm{DT}} / N=40-120 \mathrm{Td}$, while the relative ion mobilities at the same effective temperatures are given in dependence of the effective temperature. $\mathrm{In}$ b) and c) the drift fields of the labeled substances are adjusted to reach the same effective temperature as the unlabeled substances. All other experimental parameters are listed in Table 1. The error bars represent the standard deviation from three individual measurements of the same sample. The horizontal lines characterize the theoretical relative ion mobilities calculated using eq. 3 .

The relative ion mobilities can be obtained by forming the quotient of the reduced ion mobilities at the same reduced drift fields. The theoretical relative ion mobilities summarized in Table 2 for all substances investigated in this work are calculated with eq. 3 assuming equal collision cross sections and effective temperatures. As can be seen in Figure 3 a), the relative ion mobilities $K_{\mathrm{r}}$ at the same reduced drift fields of the product ions of toluene and toluene- ${ }^{13} \mathrm{C}_{7}$ have smaller values than the theoretical relative ion mobilities at a reduced drift field of $E_{\mathrm{DT}} / N=40 \mathrm{Td}$, while they increase with increasing reduced drift fields. However, the relative ion mobilities at the same reduced drift fields decrease above $E_{\mathrm{DT}} / N=80 \mathrm{Td}$, so that at $E_{\mathrm{DT}} / N=120 \mathrm{Td}$ they deviate from the theoretical relative ion mobilities with about $0.05 \%$. At high reduced drift fields, the effective temperatures of the isotopologues can differ significantly due to different energies obtained from the electric field. Thus, the differences between the observed and theoretical relative ion mobilities at high reduced drift fields probably result from the different effective temperatures.

Table 2. Investigated substances and theoretical relative ion mobilities $K_{\mathrm{r}}$ '. The data originate from eq. 3 assuming equal effective temperatures and collision cross sections.

\begin{tabular}{lc}
\multicolumn{1}{c}{ name of the compounds } & theoretical $K_{\mathrm{r}}$ \\
directly ionized toluene/toluene- $-{ }^{13} \mathrm{C}_{7}$ & 1.0084 \\
protonated toluene/toluene- ${ }^{13} \mathrm{C}_{7}$ & 1.0082 \\
directly ionized benzene/benzene $-{ }^{13} \mathrm{C}_{6}$ & 1.0096 \\
protonated benzene/benzene- ${ }^{13} \mathrm{C}_{6}$ & 1.0094 \\
protonated acetone/acetone- $-{ }^{13} \mathrm{C}_{3}$ & 1.0079 \\
protonated acetonitrile/acetonitrile- ${ }^{13} \mathrm{C}_{2}$ & 1.0092
\end{tabular}

The calculation of the theoretical relative ion mobilities using eq. 3 assumes equal effective temperatures of the isotopologues. To make a proper comparison between theoretical and observed relative ion mobilities, the ions need the same effective temperatures, so that their ion mobilities should only differ due to different reduced masses ${ }^{17}$. The effective temperatures of the isotopologues can be calculated according to eq. 4. using the determined reduced ion mobilities and the reduced electric fields. Then the reduced electric field $E_{\mathrm{B}} / N$ is determined at which isotopologue $\mathrm{B}$ has the same effective temperature as isotopologue $\mathrm{A}$ at a set reduced electric field $E_{\mathrm{A}} / N$, which is the case when both ions have the same drift velocity. The relative ion mobility $K_{\mathrm{r}}$ ' at the same effective temperatures is then given as the quotient of the reduced ion mobilities of ion $\mathrm{A}$ at the set reduced electric field $E_{\mathrm{A}} / N$ and of ion $\mathrm{B}$ at the adapted reduced electric field $E_{\mathrm{B}} / N$ according to eq. 6 . 


$$
K_{r}{ }^{\prime}=\frac{K_{0, \mathrm{~A}}\left(\frac{E_{\mathrm{A}}}{N}\right)}{K_{0, \mathrm{~B}}\left(\frac{E_{\mathrm{B}}}{N}\right)}
$$

Thus, the isotopologues are not compared at the same reduced electric field, but at the same effective temperature, allowing an independent investigation of the influence of the ion mass on the relative ion mobility. After dissociation of all clusters at high reduced electric fields, the relative ion mobilities at the same effective temperatures should remain constant and agree with theoretical relative ion mobilities.

The relative ion mobilities at same effective temperatures of the product ions of toluene and toluene- ${ }^{13} \mathrm{C}_{7}$ shown in Figure $3 \mathrm{~b}$ ) also have smaller values than the theoretical relative ion mobilities at low effective temperatures of about $T_{\text {eff }}=370 \mathrm{~K}$, while they increase with increasing effective temperatures. Remarkably, even if only elastic collisions are considered, the relative ion mobilities at the same effective temperatures remain constant at high effective temperatures above $T_{\text {eff }}=550-600 \mathrm{~K}$ and already agree well with theoretical relative ion mobilities deviating only by less than $0.02 \%$. The small offset between the theoretical and the observed relative ion mobilities may result from remaining differences in the effective temperatures due to energy loss caused by excitations of internal energy states of the neutral molecules. To ensure that no mobility shift caused by different effective temperatures remains, the dimensionless inelastic energy loss factor $\xi$ introduced by Viehland to account for the fractional energy loss at inelastic collisions is also used to calculate the effective temperatures. As shown in Figure $3 \mathrm{c}$ ), implementing an inelastic energy loss factor of $\xi=0.01$ and $\xi=0.025$ for the directly ionized and the protonated monomer of toluene shifts the relative ion mobilities at the same effective temperature towards larger values. This further improves the agreement with the theoretical relative ion mobilities, indicating that the previous deviation of less than $0.02 \%$ was still caused by different effective temperatures. A positive inelastic energy loss factor $\xi$ agrees with expectations, since it physically corresponds to an energy loss caused by inelastic collisions, resulting in a decreasing effective temperature.

The relative ion mobilities for the remaining substances in Table 2 are also investigated. The corresponding ion mobility spectra and the resulting reduced ion mobilities of the occurring product ions, as well as their mass spectra obtained with the HiKE-IMS-MS can be found in the Supporting Information. For benzene, two ion species with $\mathrm{m} / \mathrm{z}=78$ and $\mathrm{m} / \mathrm{z}=79$ can be detected in the HiKE-IMSMS, which correspond to the directly ionized and the protonated monomer, respectively. In addition, acetone and acetonitrile show ion species with $\mathrm{m} / \mathrm{z}=59$ and $\mathrm{m} / \mathrm{z}=42$, respectively, corresponding to their protonated monomers. 

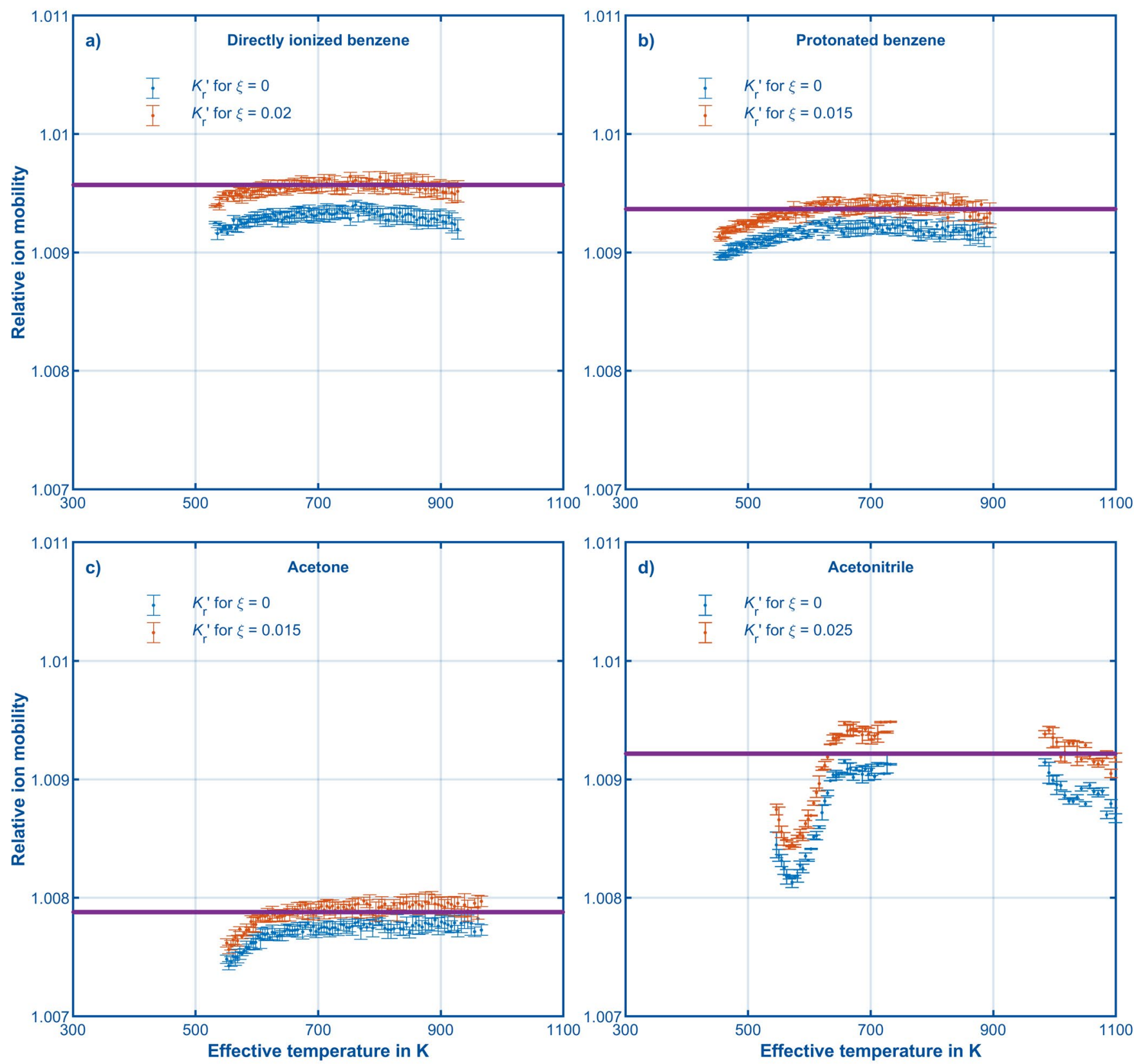

Figure 4. Relative ion mobilities $K_{\mathrm{r}}$ ' at the same effective temperatures a) for the directly ionized monomers and b) for the protonated monomers of benzene and benzene- $\left.-{ }^{13} \mathrm{C}_{6}, c\right)$ for the protonated monomers of acetone and acetone- ${ }^{13} \mathrm{C}_{3}$ and $\mathrm{d}$ ) for the protonated monomers of acetonitrile and acetonitrile- ${ }^{13} \mathrm{C}_{2}$ in nitrogen at various effective temperatures and a reduced reaction field of $E_{\mathrm{RT}} / N=70 \mathrm{Td}$. All other experimental parameters are listed in Table 1. The drift fields of the labeled substances are adjusted as explained above to reach the same effective temperature as the unlabeled substances. The error bars represent the standard deviation from three individual measurements of the same sample. The horizontal lines characterize the theoretical relative ion mobilities calculated using eq. 3 . Note that acetonitrile could not be investigated at effective temperatures of about $T_{\text {eff }}=720-980 \mathrm{~K}$, since it was overlapped by the RIP due to similar reduced ion mobilities.

Benzene, acetone and acetonitrile have higher effective temperatures than toluene at the same reduced drift field due to their higher reduced ion mobilities. However, Figure 4 shows that their relative ion mobilities at the same effective temperature behave similar to those of toluene and toluene- ${ }^{13} \mathrm{C}_{7}$. Up to an effective temperature of about $T_{\text {eff }}=550-600 \mathrm{~K}$, the relative ion mobilities at the same effective temperatures slightly increase until they remain almost constant when the effective temperature is further increased. The small offset between the observed and theoretical relative ion mobilities at effective temperatures above $T_{\text {eff }}=600 \mathrm{~K}$ is mitigated again by considering the influence of inelastic collisions on the effective temperature using the inelastic energy loss factor $\xi$.

For all substances, the relative mobilities at the same effective temperatures have smaller values than the theoretical relative ion mobilities at low effective temperatures. Under these conditions, the analyte ions can form clusters with neutral molecules resulting in an increasing ion mass. Therefore, the relative ion mobilities decrease due to the decreasing differences in the reduced masses. For effective temperatures of more than about $T_{\text {eff }}=550-600 \mathrm{~K}$ these clusters seem to be dissociated for all substances, so that at higher effective temperatures the relative ion mobilities at the same effective temperatures remain constant and agree well with theoretical values. This again shows one advantage of using a HiKE-IMS. The ion masses only agree with the expected values when clusters dissociate at high effective temperatures, allowing an accurate comparison between observed and theoretical relative ion mobilities. The agreement between measurement and theory also indicates that in these investigations all influences on the relative ion mobility are minimized except the influence of the ion mass. 
The previous results can be very useful to identify possible isotopologues. Since many elements have several stable isotopes, many compounds can occur in significant amounts in several natural isotopologues. Both the relative ion mobility and the ratio of the peak amplitudes of the ions should be known, as the latter should be proportional to the natural abundance of the ions. Here, three chlorinated hydrocarbons, namely trichloromethane (TCM), 1,1,2 - trichloroethane (1,1,2 - TCE) and 1,2,3 - trichloropropane (1,2,3 - TCP) are investigated, since they each contain three chlorine atoms, which in turn occurs in two stable isotopes ${ }^{35} \mathrm{Cl}$ and ${ }^{37} \mathrm{Cl}$ with a natural abundance of $75.76 \%$ and $24.24 \%$ respectively ${ }^{31}$.

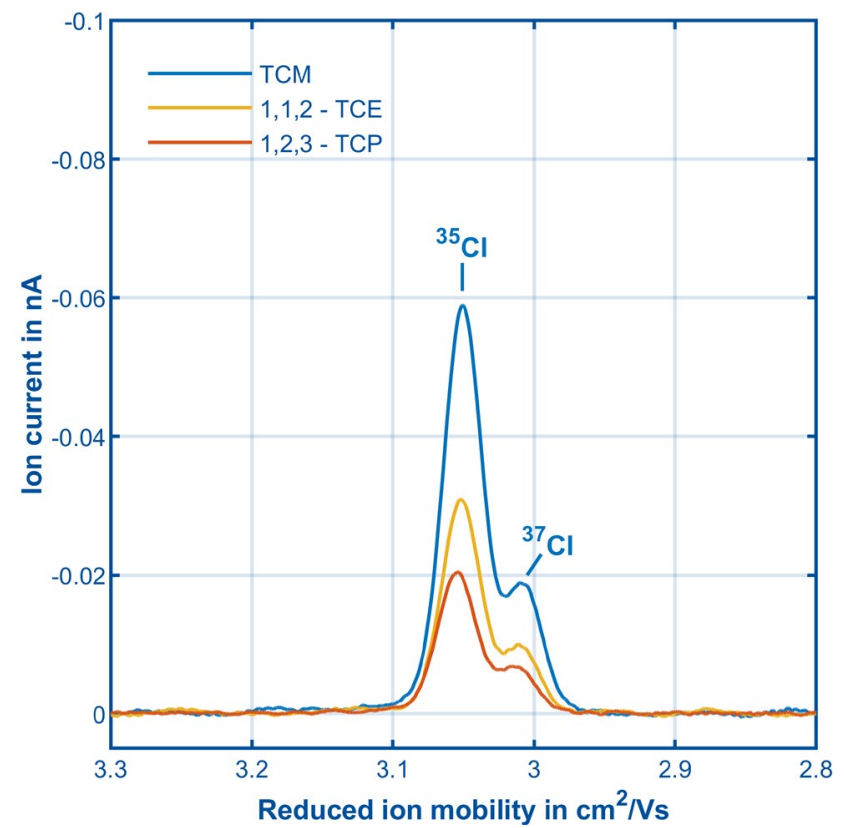

Figure 5. Ion mobility spectra of $1 \mathrm{ppm}_{\mathrm{v}}$ trichloromethane (TCM), $1 \mathrm{ppm}_{\mathrm{v}}$ 1,1,2-trichloroethane $(1,1,2-\mathrm{TCE})$ and $1 \mathrm{ppm} \mathrm{v}$ $1,2,3$ - trichloropropane $(1,2,3-\mathrm{TCP})$ in nitrogen in the negative polarity at a reduced reaction field of $E_{\mathrm{RT}} / N=60 \mathrm{Td}$ and a reduced drift field of $E_{\mathrm{DT}} / N=50 \mathrm{Td}$. All other experimental parameters are listed in Table 1 with voltages in reversed polarity.

The relative ion mobilities of the isotopologues, which can occur in the positive polarity, are too low for proper separation of the ions with the current HiKE-IMS resolving power. However, two separable peaks with high reduced ion mobilities of $K_{0,1}=3.057 \mathrm{~cm}^{2} / \mathrm{Vs}$ and $K_{0,2}=3.014 \mathrm{~cm}^{2} / \mathrm{Vs}$ at $E_{\mathrm{DT}} / N=50 \mathrm{Td}$ can be detected in the negative polarity, although they are not baseline resolved. Since no reactant ions are present in nitrogen in the negative polarity, these ions may presumably be formed by fragmentation processes of positive ions or by the electrons emitted from the corona discharge. However, the origin of these ions could not be clarified yet. Nevertheless, due to the high reduced ion mobilities and their small mobility shift it is assumed that these are the two stable chlorine isotopes ${ }^{35} \mathrm{Cl}$ and ${ }^{37} \mathrm{Cl}$. The theoretical relative ion mobility for possible ions ${ }^{35} \mathrm{Cl}^{-}$and ${ }^{37} \mathrm{Cl}^{-}$is $K_{\mathrm{r}}=1.0122$ and their relative peak amplitude should correspond to the ratio of their natural abundances of about $0.7576 / 0.2424 \approx 3.16$. As can be seen in Figure 5 , the ratios of the peak amplitudes are in the range of this expected value. However, the ratio of the peak amplitudes should be considered with care, as it can differ from the expected value, e.g. when the isotopic composition of a substance does not correspond to the natural abundance of the isotopes.

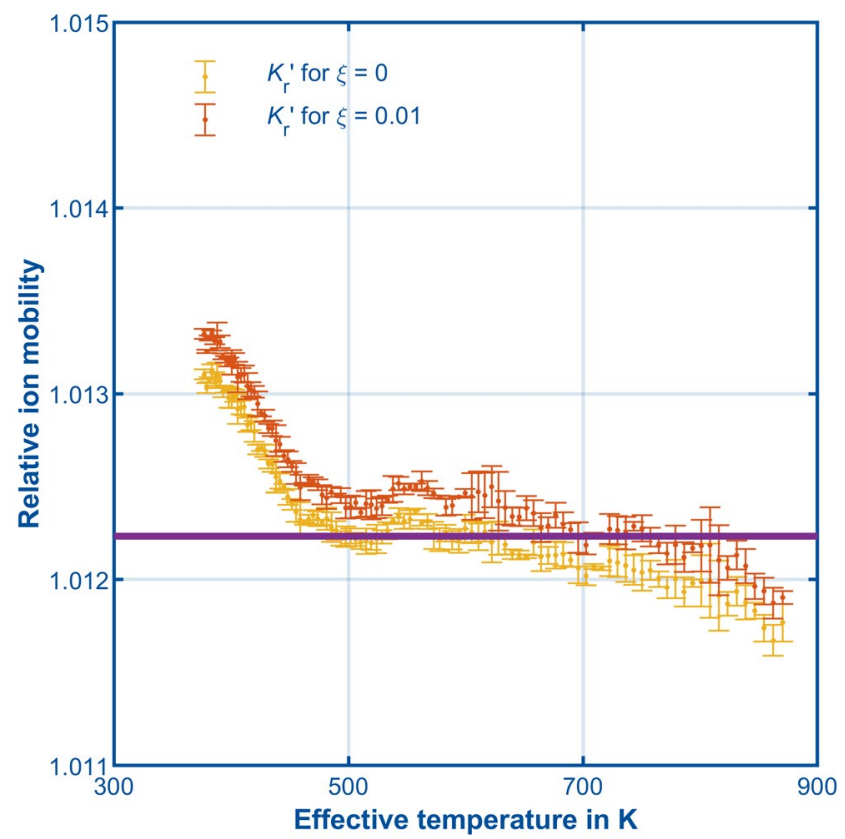

Figure 6. Relative ion mobilities $K_{\mathrm{r}}$ ' at the same effective temperatures for the ${ }^{35} \mathrm{Cl}^{-}$and ${ }^{37} \mathrm{Cl}^{-}$-fragments of $1,1,2$-tricholoroethane $(1,1,2-\mathrm{TCE})$ in nitrogen at various effective temperatures and a reduced reaction field of $E_{\mathrm{RT}} / N=60 \mathrm{Td}$. All other experimental parameters are listed in Table 1 with voltages in reversed polarity. The reduced drift fields of the labeled substances are adjusted as explained above to reach the same 
effective temperature as the unlabeled substances. The error bars represent the standard deviation from three individual measurements of the same sample. The horizontal line characterizes the theoretical relative ion mobilities calculated using eq. 3 .

The relative ion mobilities of the product ions of all three substances show a similar behavior, thus Figure 6 only shows the relative ion mobilities of the product ions of 1,1,2 - TCE for better visibility. Again, the relative ion mobilities at the same effective temperatures agree quite well with the theoretical relative ion mobilities with deviations of less than $0.05 \%$ and remain almost constant for effective temperatures above $T_{\text {eff }}=500 \mathrm{~K}$. Since the relative ion mobilities at the same effective temperatures agree well with the theoretical relative ion mobility of the two chlorine isotopes and the ratio of the peak amplitudes corresponds to the natural abundance of the isotopes, the two detected ion species are most likely ${ }^{35} \mathrm{Cl}^{-}$and ${ }^{37} \mathrm{Cl}^{-}$-fragments of the chlorinated substances. Thus, investigating the relative ion mobility and the ratio of the peak amplitudes of possible isotopologues can help identifying unknown ions in HiKE-IMS.

\section{CONCLUSION}

In this work, the relative ion mobility of several organic compounds and their ${ }^{13} \mathrm{C}$-labelled isotopologues was investigated at various reduced drift fields in a HiKE-IMS. The results show that the relative ion mobility of isotopologues depends on the reduced masses of the ions and neutral molecules and on an additional mobility shift occurring at high reduced electric fields due to the growing differences in the effective temperatures of the ions. Thus, heating effects have to be considered, when the mobility shift of isotopologues is investigated under high field conditions. However, if the isotopologues are compared at the same effective temperature, their relative ion mobility agrees well with the relative ion mobility predicted by the theory of ion mobility.

To further investigate the influence of the effective temperature on the reduced ion mobility and thus the relative ion mobility, it might be useful to investigate the mobility shift of monatomic isotopologues in a monatomic drift gas, since no energy loss due to inelastic collisions should occur under such conditions. Thus, in order to determine the relative ion mobility at the same effective temperature, it would be unnecessary to implement the inelastic energy loss factor $\xi$. In addition, no exact knowledge of the masses of the isotopologues is necessary for the determination of the effective temperatures, which is especially useful when the ions are unknown or when the ions form clusters with neutral molecules at low reduced electric fields.

The findings of this work can be useful, for example, when using isotopologues as markers in ion mobility experiments. To be able to investigate the relative ion mobilities of heavier ions and their isotopologues, the separation capability of the HiKE-IMS devices has to be further improved.

\section{AUTHOR INFORMATION}

\section{Corresponding Author}

*Fax: +49 5117623917

E-mail: schaefer@geml.uni-hannover.de

\section{Author Contributions}

C.S. and M.A. performed the experiments. C.S. and A.T.K. performed the data analysis. S.Z. and A.T.K. gave scientific and conceptual advice. S.Z. supervised the research project. All authors contributed to discussions and the manuscript.

\section{Notes}

The authors declare no competing financial interest.

\section{ACKNOWLEDGEMENTS}

Funded by the Deutsche Forschungsgemeinschaft (DFG, German Research Foundation) - 318063177.

\section{Supporting Information.}

Ion mobility spectra of reactant ions and isotopologues

Mass Spectra of reactant ions and isotopologues

Reduced ion mobilities of isotopologues

\section{REFERENCES}

(1) Hill, H. H.; Siems, W. F.; St. Louis, Robert H. Ion mobility spectrometry. Anal. Chem. 1990, 62, 1201A-1209A.

(2) Jiang, W.; Robinson, R. A.S. Ion Mobility-Mass Spectrometry (Encyclopedia of Analytical Chemistry); John Wiley \& Sons, Ltd: Chichester, UK, 2006.

(3) Lapthorn, C.; Pullen, F.; Chowdhry, B. Z. Ion mobility spectrometry-mass spectrometry (IMS-MS) of small molecules: separating and assigning structures to ions. Mass Spectrom Rev 2013, 32, 43-71.

(4) Langevin, M. P. Une formule fondamentale de théorie cinétique. Ann. Chim. Phys. 1905, 5, 245-288.

(5) Mason, E. A.; McDaniel, E. W. Transport Properties of lons in Gases; Wiley-VCH Verlag GmbH \& Co. KGaA: Weinheim, FRG, 1988.

(6) Viehland, L. A. Gaseous Ion Mobility, Diffusion, and Reaction; Springer Series on Atomic, Optical, and Plasma Physics 105; Springer International Publishing: Cham, 2018.

(7) Viehland, L. A.; Mason, E.A. Gaseous ion mobility and diffusion in electric fields of arbitrary strength. Annals of Physics 1978, 110, $287-328$.

(8) Viehland, L. A.; Fahey, D. W. The mobilities of NO -3 , NO -2 , NO + , and Cl - in N 2 : A measure of inelastic energy loss. J. Chem. Phys. 1983, 78, 435-441.

(9) Louis, Robert H. St.; Hill, H.; Eiceman, G. A. Ion Mobility Spectrometry in Analytical Chemistry. Critical Reviews in Analytical Chemistry 1990, 21, 321-355.

(10) Eiceman, G. A. Advances in Ion Mobility Spectrometry: 1980-1990. Critical Reviews in Analytical Chemistry 1991, 22, 471-490.

(11) Baumbach, J. I.; Eiceman, G. A. Ion Mobility Spectrometry: Arriving On Site and Moving Beyond a Low Profile. appl spectrosc 1999, 53, 338355.

(12) Tabrizchi, M.; Rouholahnejad, F. Comparing the effect of pressure and temperature on ion mobilities. J. Phys. D: Appl. Phys. 2005, 38, 857862. 
(13) Krylov, E. V.; Nazarov, E. G. Electric field dependence of the ion mobility. Int. J. Mass Spectrom. 2009, 285, 149-156.

(14) Gouw, J. de; Warneke, C.; Karl, T.; Eerdekens, G.; van der Veen, Carina; Fall, R. Sensitivity and specificity of atmospheric trace gas detection by proton-transfer-reaction mass spectrometry. Int. J. Mass Spectrom. 2003, 223-224, 365-382.

(15) Wannier, G. H. Motion of Gaseous lons in Strong Electric Fields. Bell Syst. Tech. J. 1953, 32, 170-254.

(16) Viehland, L. A.; Lin, S. L.; Mason, E. A. Kinetic theory of drift-tube experiments with polyatomic species. Chem. Phys. 1981, 54, 341-364.

(17) Viehland, L. A. Mobilities of isotopic ions in gases. Int. J. Ion Mobil. Spec. 2016, 19, 11-14.

(18) Kirk, A. T.; Raddatz, C.-R.; Zimmermann, S. Separation of Isotopologues in Ultra-High-Resolution lon Mobility Spectrometry. Anal. Chem. 2017, $89,1509-1515$.

(19) Valentine, S. J.; Clemmer, D. E. Treatise on the measurement of molecular masses with ion mobility spectrometry. Anal. Chem. 2009, 81, $5876-5880$.

(20) Raddatz, C.-R.; Allers, M.; Kirk, A. T.; Zimmermann, S. Acetone and perdeuterated acetone in UV-IMS. Int. J. Ion Mobil. Spec. 2018, $21,49-53$.

(21) Kaszycki, J. L.; Bowman, A. P.; Shvartsburg, A. A. Ion Mobility Separation of Peptide Isotopomers. J. Am. Soc. Mass Spectrom. 2016, 27, 795799.

(22) Kaszycki, J. L.; Baird, M. A.; Shvartsburg, A. A. Molecular Structure Characterization by Isotopic Splitting in Nonlinear lon Mobility Spectra. Anal. Chem. 2018, 90, 669-673.

(23) Pathak, P.; Baird, M. A.; Shvartsburg, A. A. Identification of Isomers by Multi-Dimensional Isotopic Shifts in High-Field lon Mobility Spectra. Anal. Chem. 2018, 90, 9410-9417.

(24) Shvartsburg, A. A.; Clemmer, D. E.; Smith, R. D. Isotopic effect on ion mobility and separation of isotopomers by high-field ion mobility spectrometry. Anal. Chem. 2010, 82, 8047-8051.

(25) Beck, D.; Ross, U.; Schepper, W. Isotope shift in the bulge effect of molecular scattering. Phys. Rev. A 1979, 19, $2173-2179$.

(26) Wojcik, R.; Nagy, G.; Attah, I. K.; Webb, I. K.; Garimella, S. V. B.; Weitz, K. K.; Hollerbach, A.; Monroe, M. E.; Ligare, M. R.; Nielson, F. F.; Norheim, R. V.; Renslow, R. S.; Metz, T. O.; Ibrahim, Y. M.; Smith, R. D. SLIM Ultrahigh Resolution Ion Mobility Spectrometry Separations of Isotopologues and Isotopomers Reveal Mobility Shifts due to Mass Distribution Changes. Anal. Chem. 2019, 91, 11952-11962.

(27) Allers, M.; Kirk, A. T.; Roßbitzky, N. von; Erdogdu, D.; Hillen, R.; Wissdorf, W.; Benter, T.; Zimmermann, S. Analyzing Positive Reactant lons in High Kinetic Energy lon Mobility Spectrometry (HiKE-IMS) by HiKE-IMS-MS. J. Am. Soc. Mass Spectrom. 2020, 31, 812-821.

(28) Kirk, A. T.; Grube, D.; Kobelt, T.; Wendt, C.; Zimmermann, S. A High Resolution High Kinetic Energy lon Mobility Spectrometer Based on a LowDiscrimination Tristate Ion Shutter. Anal. Chem. 2018, 90, 5603-5611.

(29) Allers, M.; Kirk, A. T.; Timke, B.; Erdogdu, D.; Wissdorf, W.; Benter, T.; Zimmermann, S. Negative Reactant lon Formation in High Kinetic Energy Ion Mobility Spectrometry (HiKE-IMS). J. Am. Soc. Mass Spectrom. 2020, Just accepted.

(30) Allers, M.; Kirk, A. T.; Eckermann, M.; Schaefer, C.; Erdogdu, D.; Wissdorf, W.; Benter, T.; Zimmermann, S. Positive Reactant lon Formation in High Kinetic Energy Ion Mobility Spectrometry (HiKE-IMS). J. Am. Soc. Mass Spectrom. 2020, 31, 1291-1301.

(31) Laeter, J. R. de; Böhlke, J. K.; Bièvre, P. de; Hidaka, H.; Peiser, H. S.; Rosman, K. J. R.; Taylor, P. D. P. Errata: Atomic weights of the elements: Review 2000 (IUPAC Technical Report). Pure and Applied Chemistry 2009, 81, 1535-1536. 
For Table of Contents Use Only

Ion Mobility Shift of Isotopologues in a High Kinetic Energy Ion Mobility Spectrometer (HiKE-IMS) at Elevated Effective Temperatures

Christoph Schaefer*, Ansgar T. Kirk, Maria Allers, Stefan Zimmermann

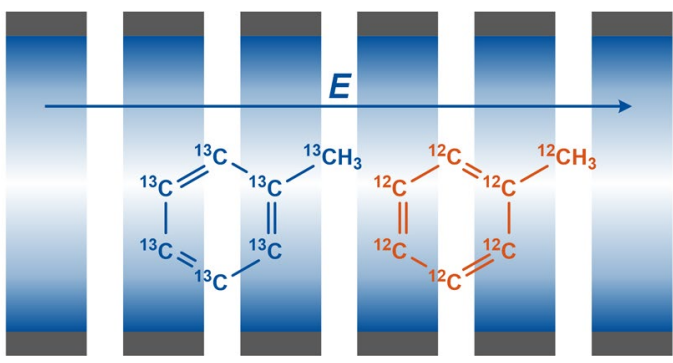

